Pacific Journal of Mathematic 


\section{EXTENSIONS OF \\ HARMONIC AND ANALYTIC FUNCTIONS}

\section{Sheldon Axler ANd Allen Shields}

Allen Shields died on 16 September 1989. This paper is dedicated to him by the first author, with many fond memories of our joint work together.

This paper studies the extensions of harmonic and analytic functions defined on the unit disk to continuous functions defined on a certain compactification of the disk.

Introduction. This introduction will present a quick survey of our results; the complete definitions necessary to state these results precisely are given in later sections.

Let $H^{\infty}$ denote the algebra of bounded analytic functions on the open unit disk in the complex plane. The maximal ideal space of $H^{\infty}$ is denoted by $M$. We can think of the open unit disk as a dense subset of $M$. Carl Sundberg [11] proved that every function in BMO extends to a continuous function from $M$ into the Riemann sphere; he also described several properties of these extensions. Sundberg was working in the context of functions of several real variables. In the next section of this paper we take advantage of the tools offered by analytic function theory to give considerably simpler proofs (in the context of one complex variable) of Sundberg's results about extensions of BMO functions.

In the section of this paper on nontangential limits, we prove that a function on the disk that has a continuous extension to a small subset of $M$ must have a nontangential limit at almost every point of the unit circle. We then use this result to produce a class of functions in the little Bloch space that cannot be extended to. be continuous functions from this small subset of $M$ to the Riemann sphere.

The section of this paper dealing with cluster sets and essential ranges shows how those sets can be computed from the appropriate continuous extensions. We use these results to give a new proof of Joel Shapiro's theorem [10] that for every function in VMO, the essential range equals the cluster set.

The final section of the paper discusses some open questions. 
We thank Peter Lappan, Joel Shapiro, and Carl Sundberg for helpful discussions.

Extensions of BMO functions. Recall that $H^{\infty}$ denotes the Banach algebra of bounded analytic functions on the unit disk $D$ and that $M$ denotes the maximal ideal space of $H^{\infty}$. Thus $M$ is the set of multiplicative linear maps from $H^{\infty}$ onto the complex numbers $\mathbf{C}$. With the usual topology (the weak-star topology that $M$ inherits as a subset of the dual of $\left.H^{\infty}\right), M$ becomes a compact Hausdorff space.

We will freely think of the disk $D$ as a subset of $M$ by identifying each point of the disk with the multiplicative linear functional of point evaluation. The topology that $D$ inherits as a subset of $M$ coincides with the usual topology on $D$.

Each $H^{\infty}$ function (thought of as a function from $D$ to $\mathbf{C}$ ) extends, via the Gelfand transform, to a continuous complex-valued function defined on $M$. A bounded real-valued harmonic function on $D$ is not necessarily the real part of an $H^{\infty}$ function. Nevertheless, if $u$ is a bounded real-valued harmonic function on $D$, then $u$ extends to a continuous function from $M$ to the reals $\mathbf{R}$. To see this, let $\tilde{u}$ denote the harmonic conjugate of $u$, so $\tilde{u}$ is the unique real-valued harmonic function on $D$ such that $u+i \tilde{u}$ is analytic on $D$ and $\tilde{u}(0)=0$. Obviously

$$
u(z)=\log \left|e^{u(z)+i \tilde{u}(z)}\right|
$$

for all $z \in D$. Thus $\log \left|\varphi\left(e^{u+i \tilde{u}}\right)\right|$ is a function (of $\varphi$ ) on $M$ that agrees with $u$ on $D$, and clearly this function is continuous on $M$. Thus we have proved the following useful lemma, whose proof we have taken from the proof of Lemma 4.4 of [8].

LEMMA 1. Let $u$ be a bounded real-valued harmonic function on $D$. Then the function $u$ defined on $M$ by

$$
u(\varphi)=\log \left|\varphi\left(e^{u+i \tilde{u}}\right)\right|
$$

is an extension of $u$ to a continuous function from $M$ to $\mathbf{R}$.

Throughout the paper we will follow the convention established in the lemma above of using the same symbol to denote a function and its extension to a larger domain. Notice that by the Corona Theorem ([5], Chapter VIII), which states that the disk $D$ is a dense subset of $M$, any continuous function on $D$ that extends to a continuous function on $M$ has a unique such extension. 
Because $M$ is compact, an unbounded function $u: D \rightarrow \mathbf{R}$ cannot be extended to a continuous function $u: M \rightarrow \mathbf{R}$. However, if we allow the extended function to take on the values $\infty$ and $-\infty$, and give $[-\infty, \infty]$ the obvious topology, then an unbounded function $u: D \rightarrow \mathbf{R}$ might extend to a continuous function $u: M \rightarrow[-\infty, \infty]$.

Carl Sundberg ([11], Theorem 3.4) proved that every real-valued function in BMO (bounded mean oscillation) extends to a continuous function from $M$ to $[-\infty, \infty]$. In this paper, we define BMO to be the set of complex-valued harmonic functions on $D$ whose real and imaginary parts can each be written as the sum of a bounded real-valued harmonic function on $D$ and the harmonic conjugate of a bounded real-valued harmonic function on $D$. For a proof that this definition is equivalent to the usual definition, see [9], page 39.

In Theorem 2 below, we give a new proof of Sundberg's theorem by using complex analysis instead of Sundberg's techniques of truncation (Sundberg was working in several real variables rather than one complex variable). Of course, the following theorem implies that if $f$ is a complex-valued function in BMO, then $f$ can be extended to a continuous function from $M$ to the Riemann sphere $\mathbf{C} \cup\{\infty\}$ (which has the obvious topology).

Theorem 2. Let $u: D \rightarrow \mathbf{R}$ be in BMO. Then $u$ can be extended to a continuous function from $M$ to $[-\infty, \infty]$.

Proof. Every real-valued function in BMO is the sum of a bounded real-valued harmonic function and the harmonic conjugate of a bounded real-valued harmonic function. By Lemma 1, every bounded realvalued harmonic function extends to a continuous real-valued function on $M$, so we can assume that $u=\tilde{v}$, where $v$ is a bounded real-valued harmonic function on $D$.

We can assume without loss of generality that

$$
\|v\|_{\infty}<\frac{\pi}{2}
$$

where $\|v\|_{\infty}$ denotes the supremum of $|v|$ on $D$. Thus $e^{\tilde{v}-i v}$ is an analytic function mapping $D$ into the open right half-plane, which we denote by RHP. Let $F:$ RHP $\rightarrow D$ be the analytic function defined by

$$
F(z)=\frac{z-1}{z+1}
$$


Then $F \circ e^{\tilde{v}-i v}$ is a bounded analytic function, and hence $F \circ e^{\tilde{v}-i v}$ extends to a continuous function $h$ on $M$, taking values in the closed disk $\bar{D}$.

Let $F^{-1}: \bar{D} \rightarrow \overline{\mathrm{RHP}} \cup\{\infty\}$ be the continuous function defined by

$$
F^{-1}(z)=\frac{1+z}{1-z}
$$

where $\overline{\mathrm{RHP}} \cup\{\infty\}$ is given the obvious topology. Note that $F^{-1} \circ F$ is the identity on RHP, and thus $\left.\left(F^{-1} \circ h\right)\right|_{D}=e^{\tilde{v}-i v}$. The absolute value function maps $\overline{\mathrm{RHP}} \cup\{\infty\}$ continuously to $[0, \infty]$, with $\left|\left(F^{-1} \circ h\right)\right|_{D} \mid=e^{\tilde{v}}$. Finally, the logarithm function maps $[0, \infty]$ continuously to $[-\infty, \infty]$, with $\log \left|\left(F^{-1} \circ h\right)\right|_{D} \mid=\tilde{v}$; see Figure 3 .

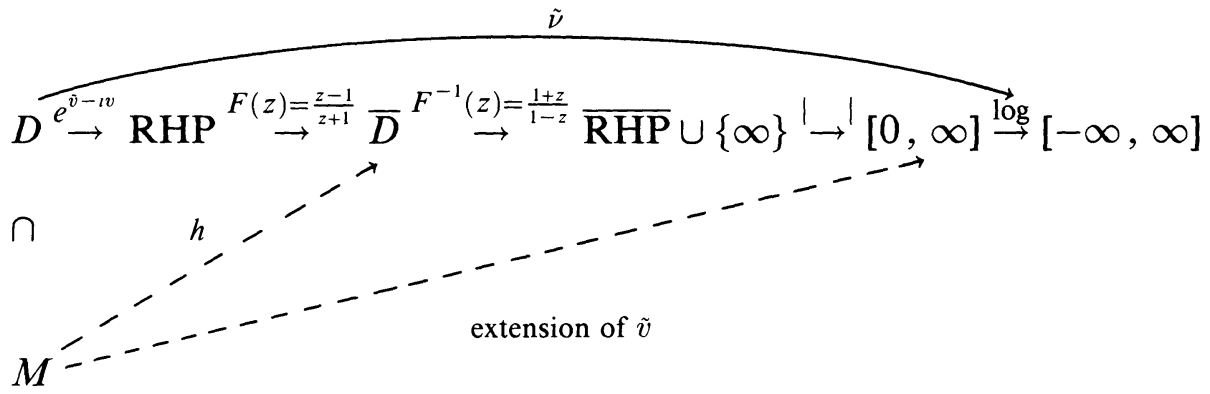

FIGURE 3

Thus the function $\log \left|\left(F^{-1} \circ h\right)\right|$ is a continuous function from $M$ into $[-\infty, \infty]$ that agrees with $\tilde{v}$ on $D$.

Because each function in $H^{\infty}$ has a unique extension to a continuous complex-valued function on $M$, we can think of $H^{\infty}$ as a closed subalgebra of $C(M)$ and apply results from the theory of function algebras. In particular, there is a smallest closed subset $X$ of $M$, called the Shilov boundary of $H^{\infty}$, such that

$$
\sup \{|f(\varphi)|: \varphi \in M\}=\sup \{|f(\varphi)|: \varphi \in X\} \quad \text { for every } f \in H^{\infty} .
$$

Because the map that sends $f$ to $\left.f\right|_{X}$ is an isometry, we see that $H^{\infty}$ is isometric to a closed subalgebra of $C(X)$. Thus by the HahnBanach Theorem and the Riesz Representation Theorem, for each $\varphi \in M$, there is a positive regular Borel measure on $X$ such that for every $f \in H^{\infty}, f(\varphi)$ can be computed by integrating $f$ against this measure. Special properties of $H^{\infty}$ (see [7], p. 182) show that this measure is unique; we denote it by $d \varphi$. We will denote the closed support of this measure by $\operatorname{supp} \varphi$, so $\operatorname{supp} \varphi$ is a closed subset of 
$X$, which is a closed subset of $M$. To summarize: For each $\varphi \in M$, there is a unique positive regular Borel measure $d \varphi$ on $X$ such that

$$
f(\varphi)=\int_{X} f d \varphi \quad \text { for every } f \in H^{\infty} .
$$

We know that if $f \in \mathrm{BMO}$, then $f$ extends to a continuous function from $M$ to the Riemann sphere $\mathbf{C} \cup\{\infty\}$, and so it is natural to ask whether this extension of $f$ satisfies the above equation. Because the extension of $f$ may take on the value $\infty$, it is not clear that the right-hand side of the above equation makes sense. However, as Sundberg proved ([11], Theorem 3.7), everything works out as nicely as possible. We reprove Sundberg's results (Propositions 4 and 6 below) using complex analysis rather than Sundberg's more complicated technique of truncation.

The following proposition clearly implies that if $u$ is a real-valued function in $\mathrm{BMO}, \varphi \in M$, and $u(\varphi)=-\infty$, then $u \equiv-\infty$ on $\operatorname{supp} \varphi$.

Proposition 4. Let $u$ be a real-valued function in $\mathrm{BMO}$, and suppose that $\varphi \in M$ is such that $u(\varphi)=\infty$. Then $u \equiv \infty$ on $\operatorname{supp} \varphi$.

Proof. Replacing $u$ by $u$ plus a bounded real-valued harmonic function will not affect either the hypothesis or the conclusion. Thus, as in the proof of Theorem 2, we can assume that $u=\tilde{v}$, where $v$ is a real-valued harmonic function on $D$ and

$$
\|v\|_{\infty}<\frac{\pi}{2}
$$

Let $h$ be as in Figure 3. From Figure 3, we see that because $\tilde{v}(\varphi)=$ $\infty$, we have $h(\varphi)=1$. On $D, h$ equals an $H^{\infty}$ function (namely $\left.F \circ e^{\tilde{v}-i v}\right)$, so

$$
1=h(\varphi)=\int_{X} h \dot{d} \varphi .
$$

On $M, h$ takes values in the closed unit disk $\bar{D}$, and the above equation says that the integral of $h$ against the probability measure $d \varphi$ is 1 . Thus $h$ must be identically 1 on $\operatorname{supp} \varphi$, which (see Figure 3 ) implies that the continuous extension of $\tilde{v}$ to $M$ is identically $\infty$ on $\operatorname{supp} \varphi$.

If $\varphi \in M$ and $f \in H^{\infty}$ is invertible in $H^{\infty}$, then Jensen's equality (see [6], Theorem 2.1) asserts that

$$
\log |\varphi(f)|=\int_{X} \log |\tau(f)| d \varphi(\tau)
$$


To prove Proposition 6, we will need Jensen's equality for another class of functions. Jensen's equality does not hold for an arbitrary outer function $f \in H^{\infty}$, but the following proposition shows that it does hold if the real part of $f$ is positive on $D$.

Proposition 5. Let $f \in H^{\infty}$ be such that $\operatorname{Re} f>0$ everywhere on $D$, and let $\varphi \in M$. Then

$$
\log |\varphi(f)|=\int_{X} \log |\tau(f)| d \varphi(\tau) .
$$

Proof. For each positive integer $n, \operatorname{Re}\left(f+\frac{1}{n}\right)>\frac{1}{n}$ on $D$, so $\left(f+\frac{1}{n}\right)$ is an invertible function in $H^{\infty}$. Thus

$$
\begin{aligned}
\log |\varphi(f)| & =\lim _{n \rightarrow \infty} \log \left|\varphi\left(f+\frac{1}{n}\right)\right| \\
& =\lim _{n \rightarrow \infty} \int_{X} \log \left|\tau\left(f+\frac{1}{n}\right)\right| d \varphi(\tau)=\int_{X} \log |\tau(f)| d \varphi(\tau),
\end{aligned}
$$

where the second equality comes from Jensen's equality for invertible functions and the last equality comes from the Monotone Convergence Theorem.

Now we can prove that the continuous extension of a BMO function can be obtained by integration against the representing measures.

Proposition 6. Let $u \in \mathrm{BMO}$, and suppose that $\varphi \in M$ is such that $u(\varphi) \in \mathbf{C}$. Then $u \in L^{1}(d \varphi)$ and

$$
u(\varphi)=\int_{X} u d \varphi
$$

Proof. We first need to prove the proposition in the case when $u$ is a bounded harmonic function on $D$. To do this, we can assume that $u$ is a bounded real-valued harmonic function on $D$. Then by Lemma 1 and Jensen's equality we have

$$
u(\varphi)=\log \left|\varphi\left(e^{u+i \tilde{u}}\right)\right|=\int_{X} \log \left|\tau\left(e^{u+i \tilde{u}}\right)\right| d \varphi(\tau)=\int_{X} u(\tau) d \varphi(\tau),
$$

as desired.

Now we no longer assume that $u$ is bounded. The paragraph above implies that replacing $u$ by $u$ plus a bounded harmonic function will not affect the conclusion. Thus, as in the proofs of Theorem 2 and 
Proposition 4, we can assume that $u=\tilde{v}$, where $v$ is a real-valued harmonic function on $D$ and

$$
\|v\|_{\infty}<\frac{\pi}{2}
$$

Let $h \in H^{\infty}$ be as in Figure 3. Then (see Figure 3)

$$
\tilde{v}(\varphi)=\log \left|\frac{1+\varphi(h)}{1-\varphi(h)}\right|=\log |1+\varphi(h)|-\log |1-\varphi(h)| .
$$

Obviously $1+\varphi(h)$ and $1-\varphi(h)$ cannot both be 0 , so the last expression makes sense and is not of the form $-\infty-(-\infty)$. Because $\tilde{v}(\varphi) \in \mathbf{C}$, neither $1+\varphi(h)$ nor $1-\varphi(h)$ can be 0 . The above equation becomes

$$
\begin{aligned}
\tilde{v}(\varphi) & =\log |\varphi(1+h)|-\log |\varphi(1-h)| \\
& =\int_{X} \log |\tau(1+h)| d \varphi(\tau)-\int_{X} \log |\tau(1-h)| d \varphi(\tau),
\end{aligned}
$$

where the last equality comes from Proposition 5 (notice that $h$ maps $D$ into $D$, so that both $1+h$ and $1-h$ have positive real parts on $D)$. Finally, the above equation becomes

$$
\tilde{v}(\varphi)=\int_{X} \log \left|\frac{1+\tau(h)}{1-\tau(h)}\right| d \varphi(\tau)=\int_{X} \tilde{v} d \varphi,
$$

where the last equality, which completes the proof, comes from Figure 3.

Nontangential limits. The Bloch space is the set of analytic functions $f$ on the disk $D$ such that

$$
\sup \left\{\left(1-|z|^{2}\right)\left|f^{\prime}(z)\right|: z \in D\right\}<\infty .
$$

The little Bloch space is the set of analytic functions $f$ on the disk $D$ such that

$$
\left(1-|z|^{2}\right) f^{\prime}(z) \rightarrow 0 \quad \text { as }|z| \uparrow 1
$$

We have seen that every BMO function can be thought of as a continuous function from $M$ to $\mathrm{C} \cup\{\infty\}$. Can this result be extended to other well-studied classes of functions? Sundberg ([11], Section 5) gave an example of a function in the Bloch space that cannot be extended to a continuous function from $M$ to $\mathbf{C} \cup\{\infty\}$. Sundberg's function is not in the little Bloch space. This raises the question of whether every function in the little Bloch space can be continuously extended to a function from $M$ to $\mathbf{C} \cup\{\infty\}$. In this section we will construct a large class of functions in the little Bloch space that do not 
have continuous extensions (with values in $\mathbf{C} \cup\{\infty\}$ ) to $M$ or even to the much smaller set $D \cup X$ (recall that $X$ is the Shilov boundary of $\left.H^{\infty}\right)$.

Our principal tool will be the following theorem about nontangential limits. When we say that a nontangential limit exists, we are allowing $\infty$ as a possible value. Statements about almost every point of $\partial D$ refer to the usual arc length Lebesgue measure on $\partial D$. Note that in this theorem $u$ is not required to be analytic or even harmonic.

THEOREM 7. Let $u$ be a continuous function on $D \cup X$ taking values in $\mathbf{C} \cup\{\infty\}$. Then $\left.u\right|_{D}$ has a nontangential limit at almost every point of $\partial D$.

Proof. We begin by considering the special case where $u$ can be extended to a continuous function from $M$ to $\mathbf{C}$. By the StoneWeierstrass Theorem, functions of the form

$$
f_{1} \overline{g_{1}}+\cdots+f_{n} \overline{g_{n}},
$$

where $f_{j}, g_{j} \in H^{\infty}$ and $n$ is an arbitrary positive integer, are dense in $C(M)$. Each such function has a nontangential limit at almost every point of $\partial D$, and hence the uniform limit of any sequence of such functions also has this property. Thus we are done in the special case under consideration.

Now consider the case where $u$ is a continuous function on $D \cup X$ that takes on values only in $\mathbf{C}$ (unlike the paragraph above, we are no longer assuming that $u$ can be continuously extended to $M)$. By the Tietz Extension Theorem, there is a function $v \in C(M)$ such that $\left.u\right|_{X}=\left.v\right|_{X}$. Notice that $u-\left.v\right|_{D \cup X}$ is a continuous complex-valued function on $D \cup X$ that is 0 on $X$. By the above paragraph, $\left.v\right|_{D}$ has nontangential limits almost everywhere on $\partial D$, so that by replacing $u$ by $u-\left.v\right|_{D \cup X}$, we can assume without loss of generality that $\left.u\right|_{X}=0$.

Let $\varepsilon$ be an arbitrary positive number. We claim that there is a positive number $\delta$ and an inner function $b$ such that

$$
\{z \in D:|b(z)|>1-\delta\} \subset\{z \in D:|u(z)|<\varepsilon\} .
$$

To see how this claim will complete the proof in the case under consideration $\left(u\right.$ is complex valued and $\left.u\right|_{X}=0$ ), let $\left(\delta_{n}\right)_{n=1}^{\infty}$, a sequence of positive numbers and $\left(b_{n}\right)_{n=1}^{\infty}$, a sequence of inner functions, be such that

$$
\left\{z \in D:\left|b_{n}(z)\right|>1-\delta_{n}\right\} \subset\left\{z \in D:|u(z)|<\frac{1}{n}\right\} .
$$


For each $n$, the function $\left|b_{n}\right|$ has nontangential limit 1 at each point of $\partial D \backslash E_{n}$, where $E_{n}$ is a subset of $\partial D$ of measure 0 . The above inclusion thus implies that $u$ has nontangential limit 0 at each point of $\partial D \backslash\left(\bigcup_{n=1}^{\infty} E_{n}\right)$, completing the proof in this case.

We now prove the claim made in the paragraph above. The set $\{\varphi \in D \cup X:|u(\varphi)|<\varepsilon\}$ is an open subset of $D \cup X$ containing $X$. Thus there is an open subset $\mathscr{O}$ of $M$ such that

$$
\{\varphi \in D \cup X:|u(\varphi)|<\varepsilon\}=\mathscr{O} \cap(D \cup X) .
$$

By the theorem on page 179 of [7], $X$ is the intersection, taken over all inner functions $b$, of the sets $\{\varphi \in M:|b(\varphi)|=1\}$. Thus

$$
\bigcap_{b \text { inner }}\{\varphi \in M:|b(\varphi)|=1\} \subset \mathscr{O} \text {. }
$$

Taking complements (in $M$ ), the above inclusion states that the compact set $M \backslash \mathscr{O}$ is contained in the union of a certain collection of open sets, and hence in the union of a finite subcollection. Taking complements again, we see that there are finitely many Blaschke products $b_{1}, \ldots, b_{n}$ such that $\left\{\varphi \in M:\left|b_{1}(\varphi)\right|=1\right\} \cap \cdots \cap\left\{\varphi \in M:\left|b_{n}(\varphi)\right|=1\right\}$ is contained in $\mathscr{O}$. Letting $b=b_{1} \cdots b_{n}$, we obtain a single inner function $b$ such that

$$
\{\varphi \in M:|b(\varphi)|=1\} \subset \mathscr{O} .
$$

Finally, $|b|$ has a maximum on the compact set $M \backslash \mathscr{O}$ which must be strictly less than 1 , so there is a positive number $\delta$ such that

$$
\{\varphi \in M:|b(\varphi)|>1-\delta\} \subset \mathscr{O},
$$

which implies that

$$
\{z \in D:|b(z)|>1-\delta\} \subset\{z \in D:|u(z)|<\varepsilon\},
$$

completing the proof of the claim.

We have now proved the theorem in the case where $u$ takes values only in $\mathbf{C}$. To complete the proof in the final case, where $u$ is a continuous function on $D \cup X$ taking values in $\mathbf{C} \cup\{\infty\}$, let $P: \mathbf{C} \cup\{\infty\} \rightarrow \mathbf{C}$ be a continuous function such that for every $w \in \mathbf{C}, P^{-1}(w)$ is a finite set. (One way to construct such a function is to think of $\mathbf{C} \cup\{\infty\}$ as the unit sphere in $\mathbf{R}^{3}$, and let $P$ map a point $(x, y, z)$ on the unit sphere in $\mathbf{R}^{3}$ to $x+i y \in \mathbf{C}$. For this choice of $P$, if $w \in D$, then $P^{-1}(w)$ contains two points; if $w \in \partial D$, then $P^{-1}(w)$ contains one point; and if $w \in \mathbf{C} \backslash D$, then $P^{-1}(w)$ contains no points.) The 
function $P \circ u$ is a continuous function on $D \cup X$ taking values in C. Thus by the earlier cases, $\left.P \circ u\right|_{D}$ has a nontangential limit at almost every point of $\partial D$.

Consider a point $\lambda \in \partial D$ at which $\left.P \circ u\right|_{D}$ has a nontangential limit $w$. On each nontangential region based at $\lambda, u$ must be near the set $P^{-1}(w)$. However, $P^{-1}(w)$ is a finite set and each nontangential region is connected, so $u$ must actually be near only one point in $P^{-1}(w)$, which means that $\left.u\right|_{D}$ has a nontangential limit at $\lambda$.

A sequence $\left\{n_{k}\right\}$ of positive integers is called a Hadamard sequence if there exists a real number $q>1$ such that $n_{k+1} / n_{k}>q$ for every $k$. The following theorem produces a class of functions in the little Bloch space that cannot be continuously extended (with values in $\mathbf{C} \cup\{\infty\}$ ) to $D \cup X$.

THEOREM 8. Let $\left(n_{k}\right)_{k=1}^{\infty}$ be a Hadamard sequence. Let $\left(a_{k}\right)_{k=1}^{\infty}$ be a sequence of complex numbers such that

$$
a_{k} \rightarrow 0 \text { and } \sum_{k=1}^{\infty}\left|a_{k}\right|^{2}=\infty \text {. }
$$

Let

$$
f(z)=\sum_{k=1}^{\infty} a_{k} z^{n_{k}} .
$$

Then $f$ is a function in the little Bloch space that cannot be extended to a continuous function from $D \cup X$ to $\mathbf{C} \cup\{\infty\}$.

Proof. Because $\left(n_{k}\right)_{k=1}^{\infty}$ is a Hadamard sequence and $a_{k} \rightarrow 0, f$ is in the little Bloch space ([1], Theorem 2). Because $\left(n_{k}\right)_{k=1}^{\infty}$ is a Hadamard sequence and $\sum\left|a_{k}\right|^{2}=\infty$, at almost every point of $\partial D$, $f$ fails to have a finite radial limit ([12], Chapter 5, Theorem 6.4). Thus at almost every point of $\partial D, f$ fails to have a finite nontangential limit. By a theorem of Plessner and Privalov ([4], Corollary 1 to Theorem 8.1), at almost every point of $\partial D, f$ fails to have a nontangential limit (finite or infinite). Thus by Theorem 7, $f$ cannot be extended to a continuous function from $D \cup X$ to $\mathbf{C} \cup\{\infty\}$.

The theorem above seems to suggest that nothing interesting can be said about extending Bloch functions continuously to $M$. However, Leon Brown and P. M. Gauthier ([3], Theorem 4) proved that every normal function (in particular, every Bloch function) can be extended to a continuous function (with values in $\mathbf{C} \cup\{\infty\}$ ) defined on a large 
subset of $M$ (namely, the union of all the nontrivial Gleason parts of $M)$.

The cluster set and essential range. In this section we will show that the cluster set and essential range of a function in $C(M)$ can be computed from values on fibers. We will then use these results and the techniques from earlier in the paper to give a new proof of Joel Shapiro's theorem [10] that for every function in VMO, the essential range equals the cluster set.

If $u$ is a function from $D$ to $\mathrm{C} \cup\{\infty\}$ and $\lambda \in \partial D$, then the cluster set of $u$ at $\lambda$, denoted $\operatorname{cl}(u, \lambda)$, is defined to be the set of $w \in \mathbf{C} \cup\{\infty\}$ such that there is a sequence $\left(z_{n}\right)_{n=1}^{\infty}$ in $D$ with $z_{n} \rightarrow \lambda$ in $\mathbf{C}$ and $u\left(z_{n}\right) \rightarrow w$ in $\mathbf{C} \cup\{\infty\}$. Notice that if $u$ extends to a continuous function from $D \cup\{\lambda\}$ to $\mathbf{C} \cup\{\infty\}$, then $\operatorname{cl}(u, \lambda)$ is $\{u(\lambda)\}$.

For $\lambda \in \partial D$, the fiber of $M$ over $\lambda$, denoted $M_{\lambda}$, is defined by

$$
M_{\lambda}=\{\varphi \in M: \varphi(z)=\lambda\} ;
$$

here $z$ denotes the identity function on $D$.

The following proposition shows that the cluster set at a point is equal to the image on the fiber of $M$ over the point. Note that the function $u$ is not required to be analytic or harmonic

Proposition 9. Let $u$ be a continuous function from $M$ to $\mathbf{C} \cup\{\infty\}$ and let $\lambda \in \partial D$. Then $\operatorname{cl}(u, \lambda)=u\left(M_{\lambda}\right)$.

Proof. First suppose that $w \in \operatorname{cl}(u, \lambda)$. Thus there is a sequence $\left(z_{n}\right)$ in $D$ such that $z_{n} \rightarrow \lambda$ and $u\left(z_{n}\right) \rightarrow w$. By the compactness of $M$, some $\varphi \in M_{\lambda}$ is in the closure of $\left\{z_{n}\right\}$; clearly $u(\varphi)=w$. Thus $\operatorname{cl}(u, \lambda)$ is contained in $u\left(M_{\lambda}\right)$.

Now suppose that $w \in u\left(M_{\lambda}\right)$. For each positive integer $n$, let $E_{n}$ be the open unit ball on the Riemann sphere of radius $1 / n$ centered at $w$. Let

$$
\mathscr{O}_{n}=\left\{\varphi \in M: u(\varphi) \in E_{n} \text { and }|\varphi(z)-\lambda|<\frac{1}{n}\right\} .
$$

Clearly $\mathscr{O}_{n}$ is an open subset of $M$. Because $w \in u\left(M_{\lambda}\right), \mathscr{O}_{n}$ is non-empty. Thus by the Corona Theorem, each $\mathscr{O}_{n}$ contains a point $z_{n} \in D$. Clearly $z_{n} \rightarrow \lambda$ in $\mathbf{C}$ and $u\left(z_{n}\right) \rightarrow w$ in $\mathbf{C} \cup\{\infty\}$, so $w \in \operatorname{cl}(u, \lambda)$. Hence $u\left(M_{\lambda}\right)$ is contained in $\operatorname{cl}(u, \lambda)$, completing the proof. 
Suppose that $u$ is a continuous function from $M$ to $\mathbf{C} \cup\{\infty\}$. Let $u^{*}$ denote the boundary value function defined for $\lambda \in \partial D$ by

$$
u^{*}(\lambda)=\lim _{r \rightarrow 1^{-}} u(r \lambda)
$$

if this limit exists in $\mathrm{C} \cup\{\infty\}$. By Theorem 7, $u^{*}$ is defined for almost all points of $\partial D$.

We will need to consider the usual Banach algebra $L^{\infty}(\partial D)$, which consists of the bounded, Borel measurable, complex-valued functions on $\partial D$. The maximal ideal space of $L^{\infty}(\partial D)$ is denoted by $M\left(L^{\infty}(\partial D)\right)$. For $\psi \in M\left(L^{\infty}(\partial D)\right)$, define $\psi^{*} \in M$ by

$$
\psi^{*}(h)=\psi\left(h^{*}\right)
$$

for $h \in H^{\infty}$. By the theorem on page 174 of [7], the map from $M\left(L^{\infty}(\partial D)\right)$ to $M$ that takes $\psi$ to $\psi^{*}$ is a homeomorphism of $M\left(L^{\infty}(\partial D)\right)$ onto $X$.

We will need the following lemma in our proof of Proposition 11.

LemMa 10. Let $u: M \rightarrow \mathbf{C}$ be a continuous function and let $\psi \in$ $M\left(L^{\infty}(\partial D)\right)$. Then

$$
u\left(\psi^{*}\right)=\psi\left(u^{*}\right) .
$$

Proof. First consider the case where $\left.u\right|_{D}$ is a real-valued harmonic function on $D$. Then

$$
u\left(\psi^{*}\right)=\log \left|\psi^{*}\left(e^{u+i \tilde{u}}\right)\right|=\log \left|\psi\left(\left[e^{u+i \tilde{u}}\right]^{*}\right)\right|,
$$

where the first equality comes from Lemma 1 and the second equality comes from the definition of $\psi^{*}$. The theory of commutative $C^{*}$-algebras shows that for an arbitrary function $U \in L^{\infty}(\partial D)$, we have $|\psi(U)|=\psi(|U|)$ and $\psi\left(e^{U}\right)=e^{\psi(U)}$. Thus the above equation becomes

$$
u\left(\psi^{*}\right)=\log \psi\left(\left|\left[e^{u+i \tilde{u}}\right]^{*}\right|\right)=\log \psi\left(e^{u^{*}}\right)=\log e^{\psi\left(u^{*}\right)}=\psi\left(u^{*}\right),
$$

completing the proof in this case.

Now consider the set of all functions $v$ in $C(M)$ such that $v\left(\psi^{*}\right)=$ $\psi\left(v^{*}\right)$. This set is closed under addition, multiplication, and uniform limits, and by the paragraph above contains all the bounded harmonic functions on $D$. By the Stone-Weierstrass Theorem, this set must be all of $C(M)$, completing the proof.

The essential range of $u^{*}$ at $\lambda$, denoted ess $\operatorname{ran}\left(u^{*}, \lambda\right)$, is defined to be the set of $w \in \mathbf{C} \cup\{\infty\}$ such that for every open subset $E$ 
of $\mathbf{C} \cup\{\infty\}$ that contains $w$ and every open interval $I$ of $\partial D$ that contains $\lambda, I \cap\left(u^{*}\right)^{-1}(E)$ has positive Lebesgue (arc length) measure. Notice that if $u^{*}$ is continuous at $\lambda$, then ess $\operatorname{ran}\left(u^{*}, \lambda\right)$ is $\left\{u^{*}(\lambda)\right\}$.

The fiber of $X$ over $\lambda$, denoted $X_{\lambda}$, is defined by

$$
X_{\lambda}=X \cap M_{\lambda} \text {. }
$$

The following proposition shows that the essential range of the boundary value function at a point is equal to the image on the fiber of $X$ over the point. Note that the function $u$ is not required to be analytic or harmonic.

THEOREM 11. Let $u$ be a continuous function from $M$ to $\mathbf{C} \cup\{\infty\}$ and let $\lambda \in \partial D$. Then ess $\operatorname{ran}\left(u^{*}, \lambda\right)=u\left(X_{\lambda}\right)$.

Proof. First we consider the case where $u$ takes values only in $\mathbf{C}$. By the theorem on page 171 of [7],

$$
\text { ess } \operatorname{ran}\left(u^{*}, \lambda\right)=\left\{\psi\left(u^{*}\right): \psi \in M\left(L^{\infty}(\lambda D)\right) \text { and } \psi^{*} \in X_{\lambda}\right\} \text {. }
$$

Lemma 10 shows that the right side of the above equation equals $u\left(X_{\lambda}\right)$, completing the proof in the case where $u$ is complex valued.

Now suppose that $u$ takes values in $\mathbf{C} \cup\{\infty\}$. As in the proof of Theorem 7, let $P: \mathbf{C} \cup\{\infty\} \rightarrow \mathbf{C}$ be a continuous function such that for every $w \in \mathbf{C}, P^{-1}(w)$ is a finite set. Then $P \circ u \in C(M)$, and so by the paragraph above the desired results holds for $P \circ u$. Thus

$$
\begin{aligned}
P\left(\text { ess } \operatorname{ran}\left(u^{*}, \lambda\right)\right) & =\text { ess } \operatorname{ran}\left(P \circ u^{*}, \lambda\right) \\
& =\text { ess } \operatorname{ran}\left((P \circ u)^{*}, \lambda\right)=(P \circ u)\left(X_{\lambda}\right)=P\left(u\left(X_{\lambda}\right)\right) .
\end{aligned}
$$

Because the above equation holds for every function $P$ as described above, we have

$$
\text { ess } \operatorname{ran}\left(u^{*}, \lambda\right)=u\left(X_{\lambda}\right)
$$

completing the proof.

In this paper, we define VMO (vanishing mean oscillation) to be the set of complex-valued harmonic functions $f$ on $D$ such that the real and imaginary parts of $f$ can each be written as the sum of a uniformly continuous, real-valued harmonic function on $D$ and the harmonic conjugate of a uniformly continuous, real-valued harmonic function on $D$. For a proof that this definition is equivalent to the usual definition, see [9], page 49.

Joel Shapiro ([10], Corollary 2.2) proved that for a function in $\mathrm{VMO}$, the cluster set equals the essential range. Using the techniques developed above, we now give a new proof of Shapiro's theorem. 
THeOREM 12. Let $u$ be a function in VMO and let $\lambda \in \partial D$. Then

$$
\operatorname{cl}(u, \lambda)=\operatorname{ess} \operatorname{ran}\left(u^{*}, \lambda\right)
$$

Proof. By Proposition 9 and Theorem 11, we need only show that

$$
u\left(M_{\lambda}\right)=u\left(X_{\lambda}\right)
$$

Obviously $u\left(X_{\lambda}\right)$ is contained in $u\left(M_{\lambda}\right)$. To prove the reverse inclusion, let $\varphi \in M_{\lambda}$. We claim that $u$ is constant on $\operatorname{supp} \varphi$. If this claim is true, then by Propositions 4 and 6 (recall that $d \varphi$ is a probability measure), $u$ must equal $u(\varphi)$ on $\operatorname{supp} \varphi$; furthermore, $\operatorname{supp} \varphi$ is contained $X_{\lambda}$ (by the first corollary on page 188 of [7]), and so $u(\varphi) \in u\left(X_{\lambda}\right)$, as desired.

To complete the proof we must show that $u$ is constant on $\operatorname{supp} \varphi$. Every uniformly continuous function on $D$ is constant on $X_{\lambda}$ (by Theorem 11), and so we can assume without loss of generality that $u=\tilde{v}$, where $v \in C(\bar{D}), v$ is real valued and harmonic on $D$, $v(\lambda)=0$, and $\|v\|_{\infty}<\pi / 2$. Because $v(\lambda)=0$, the cluster set at $\lambda$ of the $H^{\infty}$ function

$$
\frac{e^{\tilde{v}-i v}-1}{e^{\tilde{v}-i v}+1}
$$

is contained in $\mathbf{R}$. Thus by Proposition $9, h\left(M_{\lambda}\right)$ is contained in $\mathbf{R}$, where $h$ is the function defined by Figure 3 . In particular, $h$ is real valued on $\operatorname{supp} \varphi$. The support set of any representing measure is anti-symmetric, so $h$ is constant on $\operatorname{supp} \varphi$ (see [2], page 137). From Figure 3 we can now conclude that $\tilde{v}$ is constant on $\operatorname{supp} \varphi$, completing the proof.

Questions. Which harmonic (or analytic) functions on the disk extend to continuous functions from $M$ to $\mathbf{C} \cup\{\infty\}$ ? As we have seen, the collection of such functions contains BMO. However, as Sundberg pointed out ([11], pages 766), the set of analytic functions that can be continuously extended to continuous functions from $M$ to $\mathbf{C} \cup\{\infty\}$ is closed under squaring and so is strictly larger than the set of analytic functions in BMO. We note that there is no reason to suspect that the set of functions under consideration will be closed under addition. Our guess is that a harmonic function on the disk has an extension to a continuous function from $M$ to the Riemann sphere if and only if the harmonic function has bounded mean spherical oscillation, which means that the oscillation is measured in the spherical metric rather than the Euclidean metric. 
Is there a converse to Theorem 7? Specifically, if $u$ is a continuous function on $D$ that has a nontangential limit at almost every point of $\partial D$, does $u$ have a continuous extension to $D \cup X$ ? (Note: After this paper was circulated in pre-print form, Christopher Bishop and Oleg Ivanov proved that this question has an affirmative answer.)

\section{REFERENCES}

[1] J. M. Anderson, Bloch functions: The basic theory, in Operators and Function Theory (S. C. Power, editor), D. Reidel Publishing Co., Dordrecht, 1985.

[2] Andrew Browder, Introduction to Function Algebras, W. A. Benjamin, New York, 1969.

[3] Leon Brown and P. M. Gauthier, Behavior of normal meromorphic functions on the maximal ideal space of $H^{\infty}$, Michigan Math. J., 18 (1971), 365-371.

[4] E. E. Collingwood and A. J. Lohwater, The Theory of Cluster Sets, Cambridge University Press, Cambridge, 1966.

[5] John B. Garnett, Bounded Analytic Functions, Academic Press, New York, 1981.

[6] Kenneth Hoffman, Analytic functions and logmodular Banach algebras, Acta Math., 108 (1962), 271-317.

[7] _ Banach Spaces of Analytic Functions, Prentice-Hall, Englewood Cliffs, 1962.

[8] _ Bounded analytic functions and Gleason parts, Ann. of Math., 86 (1967), 74-111.

[9] Donald Sarason, Function Theory on the Unit Circle, Virginia Polytechnic and State University, 1978.

[10] Joel H. Shapiro, Cluster set, essential range, and distance estimates in BMO, Michigan Math. J., 34 (1987), 323-336.

[11] Carl Sundberg, Truncations of BMO functions, Indiana Univ. Math. J., 33 (1984), 749-771.

[12] A. Zygmund, Trigonometric Series, Vol. 1, second edition, Cambridge University Press, Cambridge, 1968.

Received November 1, 1988. Both authors were supported in part by the National Science Foundation.

Michigan State University

EAST LANSING, MI 48824

AND

UNIVERSITY OF MICHIGAN

ANN ARBor, MI 48109 



\section{PACIFIC JOURNAL OF MATHEMATICS EDITORS}

\author{
V. S. VARAdarajaN \\ (Managing Editor) \\ University of California \\ Los Angeles, CA 90024-1555-05 \\ Herbert Clemens \\ University of Utah \\ Salt Lake City, UT 84112 \\ Thomas ENRIGHT \\ University of California, San Diego \\ La Jolla, CA 92093
}

R. FINN

Stanford University

Stanford, CA 94305

HermanN FlaschKa

University of Arizona

Tucson, AZ 85721

VAUGHan F. R. Jones

University of California

Berkeley, CA 94720

STEVEN KeRCKHOFF

Stanford University

Stanford, CA 94305
C. C. Moore University of California Berkeley, CA 94720

MARTIN SCHARLEMANN University of California Santa Barbara, CA 93106

\section{HAROLD STARK}

University of California, San Diego La Jolla, CA 92093

\section{ASSOCIATE EDITORS}
R. ARENS
E. F. BECKeNBACH
B. H. NeumanN
F. WolF
(1904-1989)
K. Yoshida (1906-1982)

\section{SUPPORTING INSTITUTIONS}

UNIVERSITY OF ARIZONA

UNIVERSITY OF BRITISH COLUMBIA

CALIFORNIA INSTITUTE OF TECHNOLOGY

UNIVERSITY OF CALIFORNIA

MONTANA STATE UNIVERSITY

UNIVERSITY OF NEVADA, RENO

NEW MEXICO STATE UNIVERSITY

OREGON STATE UNIVERSITY
UNIVERSITY OF OREGON

UNIVERSITY OF SOUTHERN CALIFORNIA

STANFORD UNIVERSITY

UNIVERSITY OF HAWAII

UNIVERSITY OF TOKYO

UNIVERSITY OF UTAH

WASHINGTON STATE UNIVERSITY

UNIVERSITY OF WASHINGTON 


\section{Pacific Journal of Mathematics}

Vol. 145, No. 1 September, 1990

Sheldon Jay Axler and Allen Lowell Shields, Extensions of harmonic and

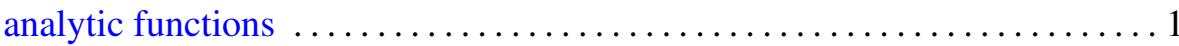

Labib Haddad and Yves Sureau, Les cogroupes et la construction de

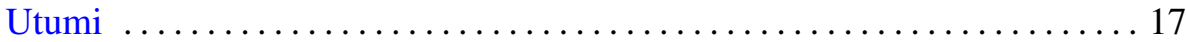

John Hutchinson, Poincaré-Sobolev and related inequalities for submanifolds of $\mathbf{R}^{N}$

Yuk Jaum Leung and Glenn E. Schober, Some coefficient problems and

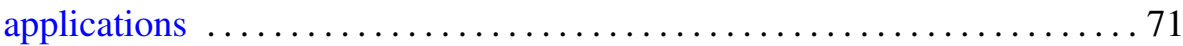

Daniel Ruberman, Seifert surfaces of knots in $S^{4} \ldots \ldots \ldots \ldots \ldots \ldots \ldots \ldots$

Joel Harold Shapiro and Carl Sundberg, Isolation amongst the

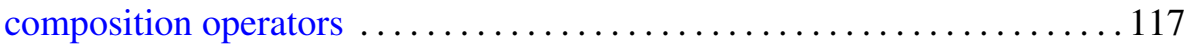

Hans Wenzl, Representations of braid groups and the quantum Yang-Baxter

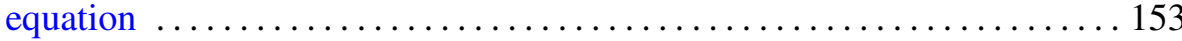

Shuang Zhang, Diagonalizing projections in multiplier algebras and in matrices over a $C^{*}$-algebra 